\title{
The Effect of Direct Communication between Emergency Physicians and Interventional Cardiologists on Door to Balloon Times in STEMI
}

\begin{abstract}
We developed an institutional protocol mandating emergency physicians to contact the interventional cardiologist directly in all cases of ST-segment elevation myocardial infarction (STEMI) and hypothesized that this would reduce door-to-balloontimes (DTBT). From January 2004 to July 2006, 208 patients with STEMI were treated with primary percutaneous coronary intervention $(\mathrm{PCl})$. A total of 144 patients were treated before implementing the new protocol ("before") and 64 patients were treated after the implementation ("after"). The DTBT was significantly reduced from $148 \pm 101 \mathrm{~min}$ to $108 \pm 56 \mathrm{~min}(p<0.05)$. While only $25 \%$ of the "before" patients received $\mathrm{PCl}$ within 90 min after arrival, $50 \%$ of the "after" patients received $\mathrm{PCl}$ within $90 \mathrm{~min}(p<0.05)$. There were no significant differences between two groups in other outcomes (postprocedural TIMI flow, mortality, subsequent stroke, heart failure, shock, reinfarction, length of stay in intensive care unit, and the total hospital length of stay). In conclusion, mandating emergency physicians to directly notify interventional cardiologists of all STEMI patients reduces DTBT.
\end{abstract}

Key Words : Myocardial infarction; Reperfusion; Electrocardiography

\author{
Min-Ji Kwak, Kyuseok Kim*, \\ Joong Eui Rhee*, Jung Ho Shin ${ }^{\star}$, \\ Gil Joon Suh, Young-Seok Jo', \\ Tae-Jin Youn', Woo-Young Chung ${ }^{\dagger}$, \\ In-Ho Chae ${ }^{\dagger}$, Dong-Ju Choi', \\ Christopher C. Lee ${ }^{\ddagger}$, and Adam J. Singer
}

Departments of Emergency Medicine ${ }^{\star}$ and Cardiology ${ }^{\dagger}$, Seoul National University Bundang Hospital, Seongnam; Department of Emergency Medicine, Seoul National University Hospital, Soeul, Korea; Department of Emergency Medicine ${ }^{\ddagger}$, Stony Brook University Hospital, NY, USA

Received: 17 January 2007 Accepted : 5 December 2007

\section{Address for correspondence \\ Kyuseok Kim, M.D. \\ Department of Emergency Medicine, Seoul National \\ University Bundang Hospital, 300 Gumi-dong, \\ Bundang-gu, Seongnam 463-707, Korea \\ Tel : +82.31-787-7579, Fax : +82.31-787-4055 \\ E-mail : dremkks@snubh.org \\ *The authors have no commercial associations or sources of support that might pose a conflict of interest.}

\section{INTRODUCTION}

Shorter time to coronary reperfusion is associated with better survival for patients with ST-segment elevation myocardial infarction (STEMI) (1). The American Heart Association and the American College of Cardiology (AHA/ ACC) have recommended that fibrinolytics be administered within $30 \mathrm{~min}$ of arrival or that percutaneous coronary intervention (PCI) be performed within 90 min of arrival in patients with acute STEMI $(2,3)$. However, in many cases door-toballoon time (DTBT) is longer than $90 \mathrm{~min}$, and recent data from the National Registry of Myocardial Infarction (NRMI) showed that the median DTBT in the United States was $185 \mathrm{~min}$ and that only $3 \%$ of patients were being mechanically reperfused within the 90-min time frame (3). Potential delays during the in-hospital evaluation period may occur at several points of the process of care (e.g., door to ECG, from ECG to decision, and from decision to PCI). Although some delays are inevitable, others may be preventable through systems and process redesign (1). In one study, they showed that emergency physician-initiated PCI reduced DTBT sig- nificantly (4).

In most hospitals in Korea, emergency physicians are required to contact an internist prior to contacting the interventional cardiologist in all cases of STEMI prior to PCI. The decision to perform PCI is then made by the interventional cardiologist. The need to first contact an internist may result in significant delays making it difficult to meet AHA/ACC guidelines. According to the AHA/ACC Guidelines for the Management of Patients With STEMI, the choice of initial management for patients with STEMI should be made by the emergency medicine physician on duty based on predetermined, institution-specific, written protocols that are a collaborative effort or partnership between various clinical services and hospitals (5). The objective of the current study was to implement a program aimed at reducing DTBT in the emergency department (ED), by requiring the emergency physician to bypass the internist and to directly communicate with the interventional cardiologists in all cases of STEMI. We hypothesized that direct contact of the ED physician with the cardiologist would reduce the DTBT in patients presenting to the ED with STEMI. 


\section{MATERIALS AND METHODS}

\section{Study settings and subjects}

A before and after study design was used to determine the effects of implementing an institutional process mandating direct communication between ED physicians and interventional cardiologists on DTBT in ED patients with STEMI undergoing PCI (Fig. 1). This study was conducted at a 950bed, urban, university-based hospital. Patients who presented to the ED with STEMI and underwent emergent PCI between January 1, 2004 and July 31, 2006 were enrolled. Our cardiac catheterization lab is available 24-hr a day, 7days a week. During weekdays (8 a.m. to 8 p.m.), all members of the catheterization team are physically present in the hospital, while on weekends and during the night on-call staffs are available within $30 \mathrm{~min}$.

\section{Data collection and outcome measures}

Demographic and clinical data were extracted from electronic medical records of all study patients. Data included age, gender, presenting symptoms and prior medical history. Accessible time segments of DTBT (door to ECG time, door to emergency physician time, door to internal medicine

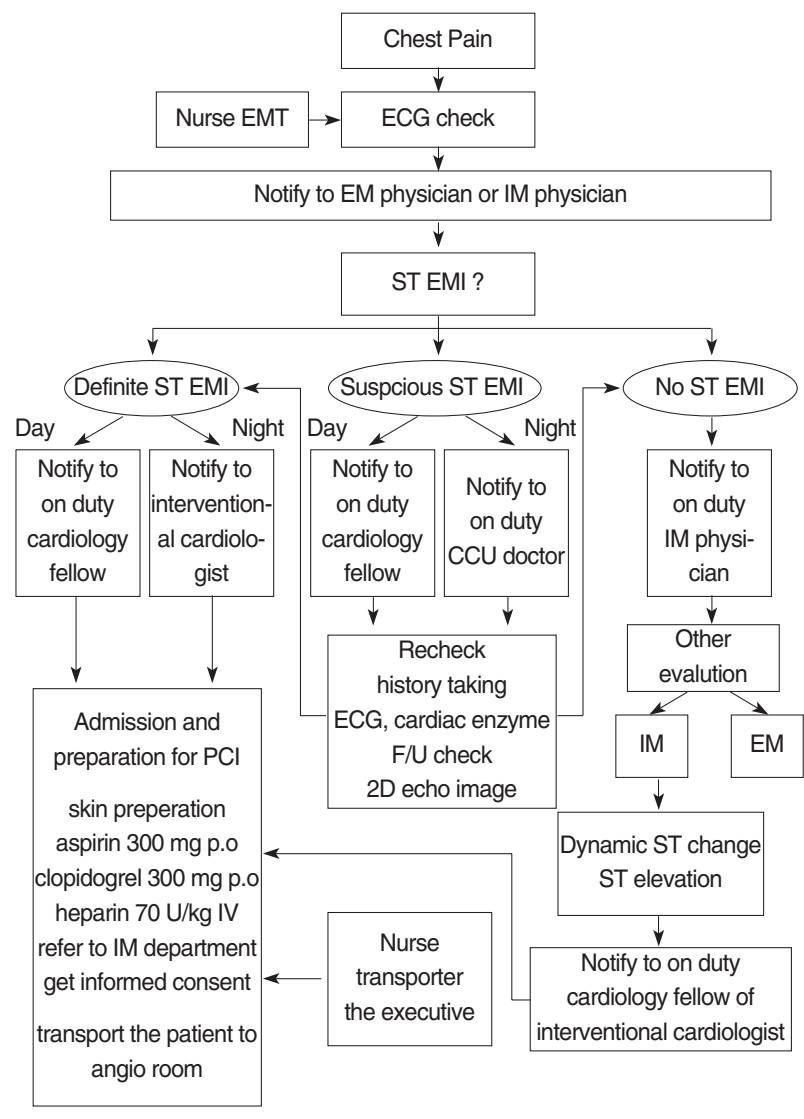

Fig. 1. Flow chart for patients with chest pain. resident time, door to arrival of catheterization laboratory time, and time from arrival of catheterization laboratory to PCI time) were acquired and compared between the 2 study groups. The primary outcomes were the time interval in min between hospital arrival and the delivery of reperfusion therapy (DTBT) and the percentage of patients undergoing PCI within 90 min of ED arrival. Secondary outcomes were postprocedural Thrombolysis in Myocardial Infarction (TIMI) flow rates, in-hospital mortality, presence of cardiogenic shock, heart failure, stroke, reinfarction and the use of an intra aortic balloon pump (IABP). We also determined the length of stay in the intensive care unit (LOS in ICU) and total duration of hospital stay (6).

\section{Statistical analysis}

Continuous data were described as means and $95 \%$ confidence intervals (CIs) and were compared with t-tests. Binomial data were described as percent frequency of occurrence and were compared with $\chi^{2}$ tests. Data were analyzed using SPSS (version 13.0) for Windows (SPSS Inc., Chicago, IL). A $p$ value of $<0.05$ was considered statistically significant.

\section{RESULTS}

During the study period, 208 patients presented with acute STEMI and underwent primary emergent PCI. Of all patients, 144 were treated before protocol implementation, and 64 patients were treated after implementing the institutional protocol mandating direct communication between ED physicians and interventional cardiologists. Both groups were similar in baseline characteristics except for the incidence of dyslipidemia (Table 1).

\section{Effect of STEMI protocol on primary outcomes}

Before implementing the institutional protocol, the mean DTBT was $148 \pm 101 \mathrm{~min}$ (Table 2). After implementing the new protocol, the mean DTBT was $108 \pm 56$ min. Thus, the implementation of the new protocol reduced the DTBT by $40 \mathrm{~min}$. The percentage of patients undergoing PCI within 90 min of arrival increased from $25 \%$ to $50 \%$ after implementing the new protocol (mean difference, 25\%) (Fig. 2). The specific time intervals comprising the DTBT in both groups are presented in Table 2 .

\section{Effect of STEMI protocol on secondary outcomes}

There were no significant differences between the two groups in any of the secondary outcomes such as the postprocedural TIMI flow rates, in-hospital mortality, or rates of subsequent stroke, heart failure, shock, and reinfarction. There were also no differences in total and ICU lengths of stay (Table 3). 
Table 1. Baseline characteristics of patients*

\begin{tabular}{|c|c|c|c|}
\hline & Before-group $(n=144)$ & After-group ( $\mathrm{n}=64)$ & $p$ value \\
\hline Age (yr) & $61.6(59.5-63.6)$ & $61.6(57.9-65.3)$ & 0.99 \\
\hline Male & $112(78.9 \% \mathrm{Cl} 72.1-85.7 \%)$ & 45 (70.3\% Cl 58.8-81.8\%) & 0.18 \\
\hline Hypertension & $67(47.2 \%$ Cl 38.9-55.5\%) & 34 (57.6\% Cl 44.6-70.6\%) & 0.18 \\
\hline Diabetes mellitus & $32(22.5 \%$ Cl 15.6-29.5\%) & $15(25.4 \% \mathrm{Cl} 14.0-36.9 \%)$ & 0.66 \\
\hline Smoking & 83 (58.5\% Cl 50.3-66.7\%) & 37 (67.8\% Cl 52.9-82.7\%) & 0.58 \\
\hline Dyslipidemia & $18(12.7 \% \mathrm{Cl} 7.1-18.2 \%)$ & $18(30.5 \% \mathrm{Cl} 18.4-42.7 \%)$ & $<0.01$ \\
\hline Killip class 4 & $17(12.0 \% \mathrm{Cl} 6.6-17.4 \%)$ & $8(14.1 \%$ Cl 5.3-22.8\%) & 0.68 \\
\hline Age $>75 \mathrm{yr}$ & 22 (15.5\% Cl 9.5-21.5\%) & $12(18.8 \% \mathrm{Cl} 8.9-28.6 \%)$ & 0.56 \\
\hline Pre-TIMl flow 0-1 & 92 (66.2\% Cl 58.2-74.2\%) & 46 (71.9\% Cl 60.6-83.2\%) & 0.42 \\
\hline IABP & $19(13.7 \%$ Cl $7.9-19.5 \%)$ & $13(22.0 \% \mathrm{Cl} 11.1-32.9 \%)$ & 0.14 \\
\hline Mean SBP (mmHg) & $132.5(127.3-137.8)$ & $134.1(126.4-141.7)$ & 0.75 \\
\hline Mean DBP $(\mathrm{mmHg})$ & $80.6(77.0-84.2)$ & $79.8(74.8-84.9)$ & 0.82 \\
\hline Mean HR (beats/min) & $72.0(68.8-75.3)$ & $75.8(70.8-80.9)$ & 0.21 \\
\hline $\mathrm{RR}(/ \mathrm{min})$ & $19.9(19.2-20.6)$ & $21.2(19.6-22.9)$ & 0.14 \\
\hline $\mathrm{BT}\left({ }^{\circ} \mathrm{C}\right)$ & $36.2(36.1-36.3)$ & $36.3(36.2-36.5)$ & 0.25 \\
\hline
\end{tabular}

${ }^{*}$ Continuous variables are expressed as mean with 95\% confidence interval $(\mathrm{Cl})$.

IABP, intra-aortic balloon pump; TIMI, thrombolysis in myocardial infarction; SBP, systolic blood pressure; DBP, diastolic blood pressure; HR, heart rate; $\mathrm{RR}$, respiration rate; $\mathrm{BT}$, body temperature.

Table 2. Comparison of DTBT segments and primary outcome*

\begin{tabular}{lccr}
\hline Mean time & $\begin{array}{c}\text { Before-group } \\
(\mathrm{n}=144)\end{array}$ & \multicolumn{1}{c}{$\begin{array}{c}\text { After-group } \\
(\mathrm{n}=64)\end{array}$} & \multicolumn{1}{c}{$\begin{array}{c}p \\
\text { value }\end{array}$} \\
\hline DTBT (min) & $148.4(131.6-165.1)$ & $107.7(93.7-121.7)$ & $<0.01$ \\
Door to ECG (min) & $5.5(4.0-6.9)$ & $6.9(4.4-9.4)$ & 0.31 \\
Door to EP (min) & $4.7(0-9.9)$ & $3.5(0-7.6)$ & 0.72 \\
Door to IM (min) & $17.3(13.3-21.4)$ & & \\
DTCT (min) & $110.9(94.3-127.5)$ & $78.3(60.4-96.2)$ & $<0.01$ \\
Cath. Lab to PCl (min) & $37.7(35.3-40.2)$ & $35.4(32.0-38.9)$ & 0.28 \\
DTBT <60 min (\%) & $2.1(0-4.5)$ & $10.9(3.1-18.8)$ & $<0.01$ \\
DTBT <90 min (\%) & $25.2(18.0-32.4)$ & $50.0(37.4-62.6)$ & $<0.01$ \\
DTCT <60 min (\%) & $14.7(8.8-20.6)$ & $40.6(28.3-53.0)$ & $<0.01$ \\
\hline
\end{tabular}

${ }^{*}$ Continuous variables are expressed as mean with $95 \%$ confidence interval (Cl).

ECG, electrocardiograph; EP, emergency physician; IM, internal medicine physician; DTBT, door-to-balloon-time; DTCT, door-to-catheterization room-time.

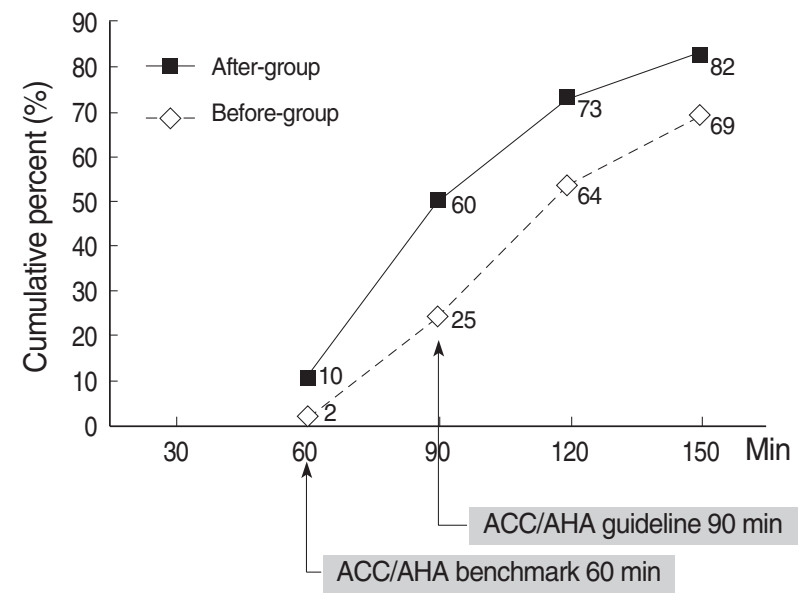

Fig. 2. Comparison of percentage of door-to-balloon-times within 60 or 90 min in STEMI patients between the two study groups.

Table 3. Comparison of secondary clinical outcomes ${ }^{\star}$

\begin{tabular}{lccc}
\hline & Before-group $(\mathrm{n}=144)$ & After-group $(\mathrm{n}=64)$ & $p$ value \\
\hline Death & $10(7.1 \% \mathrm{Cl} 2.8-11.4 \%)$ & $7(10.9 \% \mathrm{Cl} 3.1-18.8 \%)$ & 0.41 \\
Shock & $25(18.2 \% \mathrm{Cl} 11.7-24.8 \%)$ & $8(14.6 \% \mathrm{Cl} 4.6-22.6 \%)$ & 0.42 \\
CHF & $18(13.2 \% \mathrm{Cl} 7.5-19.0 \%)$ & $5(10.4 \% \mathrm{Cl} 1.5-19.4 \%)$ & 0.61 \\
Reinfarction & $2(1.5 \% \mathrm{Cl} 0-3.5 \%)$ & $1(1.7 \% \mathrm{Cl} 0-5.2 \%)$ & 0.89 \\
Stroke & $3(2.2 \% \mathrm{Cl} 0-4.7 \%)$ & $4(6.5 \% \mathrm{Cl} 0.2-12.7 \%)$ & 0.13 \\
Post-TIMl flow $\geq 3$ & $131(91.0 \% \mathrm{Cl} 86.2-95.7 \%)$ & $58(90.6 \% \mathrm{Cl} 83.3-98.0 \%)$ & 0.65 \\
EF $(\%)$ & $46.5(43.2-49.8)$ & $49.4(45.9-52.9)$ & 0.26 \\
LOS in ICU (days) & $3.4(3.0-3.7)$ & $3.4(2.6-4.2)$ & 0.99 \\
LOS in hospital (days) & $8.1(7.1-9.2)$ & $8.9(7.0-10.8)$ & 0.46 \\
\hline
\end{tabular}

${ }^{*}$ Continuous variables are expressed as mean with 95\% confidence interval (Cl).

$\mathrm{CHF}$, congestive heart failure; TIMI, thrombolysis in myocardial infarction; EF, ejection fraction; LOS, length of stay at hospital; ICU, intensive care unit stay. 


\section{Application of STEMI Protocol on day time and night time}

Although primary PCI is available for 24-hr a day and 7days a week in our institute, it is on-call at night time (from 8 a.m. to 8 p.m.). For that reason, we compared the DTBT during day time and night time in each group. The DTBT was significantly different during and after normal business hours (weekdays) in the "before" group; $124 \pm 73 \mathrm{~min}$ vs. $178 \pm 122 \mathrm{~min}(p=0.002)$. However, in the "after" group, the DTBT was not significantly different; $110 \pm 52 \mathrm{~min}$ vs. 105 $\pm 59 \min (p>0.05)$.

\section{DISCUSSION}

The evidence continues to mount supporting PCI as the strategy of choice for opening acutely occluded coronary arteries. Many institutions, however, struggle to meet the AHA/ACC 90 min bench mark for DTBT in acute STEMI (3). Barriers to timely and efficient PCI include delays introduced by the lack of a standard, institution-specific strategy $(7,8)$. Indeed, considerable time may be lost while ED physicians attempt to contact physicians who are authorized to determine the need for primary emergent PCI. The results of the current study demonstrate that direct communication between the ED physician and the interventional cardiologist reduces the DTBT by $40 \mathrm{~min}$ and increases the number of patients undergoing PCI within $90 \mathrm{~min}$ from $25 \%$ to $50 \%$.

In this study, we shortened the process of care for STEMI patients by having ED physicians bypass the on call internist and directly contacting the interventional cardiologist for all patients. Other time intervals, such as door to ECG, door to emergency physician, and time from arrival in catheterization lab to PCI were not significantly different between the two groups. Thus, we can conclude that the reduction in DTBT mainly results from bypassing the on call internist. This is in accordance with international recommendations that encourage the development of inter-disciplinary efforts and teams to improve the care of patients with acute coronary syndromes (5). Thus, this study demonstrates the feasibility of implementing such cooperative efforts in emergency medical centers in Korea and should serve as an example for other medical centers and countries. We, therefore, recommend that all emergency medical centers that perform primary PCI in Korea consider the development of institutional protocols and certainly encourage greater collaboration between ED physicians, internists and cardiologists in order to improve the care of STEMI patients in Korea.

While we reduced mean DTBT, many patients still did not meet AHA/ACC guidelines for PCI within 90 min of arrival. There are many potential factors that may have contributed to the delay in treating STEMI patients at our medical center. These may include a delay in acquiring and inter- preting ECGs, delays in patient transport to the catheterization lab, delays in activating the interventional cardiology team, delays in preparing the catheterization lab, or the unavailability of the catheterization lab due to patient overload. Additionally, efforts to reduce the delay in patient arrival to the ED should be made (9-12).

Our study had several limitations that need further discussion. First, this was not a randomized trial. Therefore, we cannot control for all potential confounding variables. Second, this study was limited to a single academic medical center with continuous availability of a cardiac catheterization lab and staff. Thus, our results may not be generalized to other institutions or settings. However, our study does serve as a feasibility study for the other emergency medical centers in Korea. Third, we did not compare the rate of "false positive" contacts (contact to interventional cardiologist without STEMI) before- and after-implementing the new protocol due to its retrospective nature. There was only one such false positive case in the after group where the intervention cardiologist did not perform cardiac catheterization. The patient complained of severe chest pain and presented with ST elevation on the ECG, but after administration of nitroglycerin, his chest pain resolved and his ECG normalized. We concluded that his chest pain was due to coronary vasospasm. It is well accepted that such "false positives" are an inevitable consequence of attempts to minimize DTBT, and Bradley et al. have reported that in the top-performing hospitals, such "false positives" rarely occur and are acceptable (9). Fourth, we could not investigate all time intervals of the DTBT due to the retrospective nature of the study. For example, we could not abstract data about the door to cardiologist-call time, or door to decision of PCI time. However, since the other time intervals of the DTBT were similar in both study groups, we can conclude that the main advantage of this protocol was the direct communication and notification from the ED physician to the interventional cardiologist. Finally, our study was too small to determine the effects of the study interventions on other more important outcomes such as mortality. Although not statistically significant, the incidence of some adverse secondary outcomes was higher in the after-group. We think this was mainly due to chance; however, another study also showed that some complications occurred more frequently in after implementing a similar protocol (4).

In conclusion, the current study demonstrates that implementation of an institutional protocol that requires ED physicians to directly contact interventional cardiologists regarding all patients with acute STEMI reduces the mean DTBT and increases the percentage of STEMI patients undergoing PCI within 90 min of arrival.

\section{REFERENCES}

1. McNamara RL, Herrin J, Bradley EH, Portnay EL, Curtis JP, Wang 
Y, Magid DJ, Blaney M, Krumholz HM. Hospital improvement in time to reperfusion in patients with acute myocardial infarction, 1999-2002. J Am Coll Cardiol 2006; 47: 45-51.

2. ECC Committee, Subcommittees and Task Forces of the American Heart Association. 2005 American Heart Association Guidelines for Cardiopulmonary Resuscitation and Emergency Cardiovascular Care. Circulation 2005; 112 (Suppl): IV1-203.

3. NRMI4 Investigators. The national registry of myocardial infarction 4 quarter data report. South San Francisco, CA: Genentech; 2003.

4. Thatcher JL, Gilseth TA, Adlis S. Improved efficiency in acute myocardial infarction care through commitment to emergency department-initiated primary PCI. J Invasive Cardiol 2003; 15: 693-8.

5. Antman EM, Anbe DT, Armstrong PW, Bates ER, Green LA, Hand M, Hochman JS, Krumholz HM, Kushner FG, Lamas GA, Mullany CJ, Ornato JP, Pearle DL, Sloan MA, Smith SC Jr, Alpert JS, Anderson JL, Faxon DP, Fuster V, Gibbons RJ, Gregoratos G, Halperin JL, Hiratzka LF, Hunt SA, Jacobs AK, Ornato JP. ACC/AHA guidelines for the management of patients with ST-elevation myocardial infarction; a report of the American College of Cardiology/American Heart Association Task Force on Practice Guidelines (Committee to revise the 1999 guidelines for the management of patients with acute myocardial infarction). J Am Coll Cardiol 2004; 44: E1-E211.

6. De Luca G, Suryapranata H, van't Hof AW, de Boer MJ, Hoorntje JC, Dambrink JH, Gosselink AT, Ottervanger JP, Zijlstra F. Prognostic assessment of patients with acute myocardial infarction treated with primary angioplasty: implications for early discharge. Cir- culation 2004; 109: 2737-43.

7. Doorey A, Patel S, Reese C, O'Connor R, Geloo N, Sutherland S, Price N, Gleasner E, Rodrigue R. Dangers of delay of initiation of either thrombolysis or primary angioplasty in acute myocardial infarction with increasing use of primary angioplasty. Am J Cardiol 1998; 81: 1173-7.

8. Thatcher JL, Gilseth TA. Experience with process improvement and outcomes analysis in acute myocardial infarction in a community hospital, 1990-1999. (Abstr) J Invas Cardiol 2000; 12: 574.

9. Bradley EH, Roumanis SA, Radford MJ, Webster TR, McNamara RL, Mattera JA, Barton BA, Berg DN, Portnay EL, Moscovitz H, Parkosewich J, Holmboe ES, Blaney M, Krumholz HM. Achieving door-to-balloon times that meet quality guidelines: how do successful hospitals do it? J Am Coll Cardiol 2005; 46: 1236-41.

10. Terkelsen CJ, Norgaard BL, Lassen JF, Gerdes JC, Ankersen JP, Romer F, Nielsen TT, Andersen HR. Telemedicine used for remote prehospital diagnosing in patients suspected of acute myocardial infarction. J Intern Med 2002; 252: 412-20.

11. Sekulic M, Hassunizadeh B, McGraw S, David S. Feasibility of early emergency room notification to improve door-to-balloon times for patients with acute ST segment elevation myocardial infarction. Catheter Cardiovasc Interv 2005; 66: 316-9.

12. Kereiakes DJ, Gibler WB, Martin LH, Pieper KS, Anderson LC. Relative importance of emergency medical system transport and the prehospital electrocardiogram on reducing hospital time delay to therapy for acute myocardial infarction: a preliminary report from the Cincinnati Heart Project. Am Heart J 1992; 123: 835-40. 